\title{
Estimación de Vitamina D en mujeres con osteopenia y osteoporosis en Cundinamarca-Colombia, por medio de extracción en fase sólida, cromatografía líquida de alta resolución y análisis multivariado
}

\author{
Magda Guevara ', Leidy Mogollón ', Antonio Iglesias ～ㄹ, Hernán Yupanqui $\quad{ }^{3}$ y \\ Antonio Bermúdez $4 *$ \\ ${ }^{1}$ Universidad Colegio Mayor de Cundinamarca, Bogotá, Colombia. - $\quad{ }^{2}$ Clínica Salud REINUN. - ${ }^{3}$ Hospital Universitario de la \\ Samaritana, Bogotá. - $\quad{ }^{4}$ Subdirección de Epidemiología, Instituto Nacional de Salud, Bogotá, D.C., Colombia. \\ *Contribución al plan de vigilancia centinela para osteoporosis que el Instituto Nacional de Salud realiza desde 1998 \\ Recibido: 03-07-03; Aceptado: 22-11-03
}

\section{RESUMEN}

Actualmente existe gran interés en el metabolismo mineral y el papel de la vitamina $\mathrm{D}_{3}$ por su importancia en el entendimiento de la patogénesis de la osteoporosis y, en consecuencia, por sus aplicaciones terapéuticas y preventivas. La cuantificación de la Vitamina D implica varios pasos previos: separación, extracción y fraccionamiento, que requieren el desarrollo de diversos métodos buscando exactitud, precisión, rapidez y versatilidad. El presente estudio tuvo como objetivo cuantificar las concentraciones séricas de los principales metabolitos de la vitamina $\mathrm{D}_{3}$ : 24-hidroxicolecalciferol, 24,25-dihidroxicolecalciferol y 1,25 dihidroxicolecalciferol expresados como vitamina $\mathrm{D}_{3}$ total en 50 pacientes con osteopenia u osteoporosis. El ensayo se realizó por un sistema de HPLC. La recuperabilidad post-extracción fue del 85\%, la sensibilidad para el 1,25 dihidroxicolecalciferol fue de $12.37 \mathrm{pg} / \mathrm{ml}$ y del colecalciferol de $25 \mathrm{pg} / \mathrm{ml}$. El promedio de Vitamina $\mathrm{D}_{3}$ total fue de $2072 \mathrm{ng} / \mathrm{ml}$ con un intervalo de 6.3 a $10600 \mathrm{ng} / \mathrm{ml}$ y se halló diferencia significativa en las medias de concentración de Vitamina D entre mujeres con DMO normal y las que tenían al menos osteopenia en cadera $(p<0.04)$; sin embargo, esta diferencia no fue significativa en pacientes en columna. Se encontró una diferencia significativa de concentraciones medias de vitamina $\mathrm{D}$ entre casos con y sin antecedente de fractura $(\mathrm{p}=0.12)$. Se discute la relación de las concentraciones de vitamina $\mathrm{D}$ con los valores de densidad mineral ósea y variables clínicas, biológicas y sociodemográficas.

Palabras clave : Vitamina $\mathrm{D}_{3}$ y metabolitos, HPLC, Cartuchos Sep-Pak, Colecalciferol, 1,25-(OH) $-\mathrm{D}_{3}$, Osteoporosis.

\begin{abstract}
Vitamin D estimate in women with osteopenia and osteoporosis in Cundinamarca, Colombia, by means of extraction in solid phase, liquid chromatography of high resolution and multivaried analysis

At present great interest in the mineral metabolism exists and the role of the vitamin $\mathrm{D}_{3}$, by its importance in the understanding of the pathogenesis of the Osteoporosis and consequently by its preventive and therapeutic applications. The quantification of the Vitamin D implies various previous steps: separation, extraction and
\end{abstract}


division that require the development of diverse methods seeking accuracy, precision, rapidity and versatility. The present study had as an objective to quantify the concentrations from serum of the main metabolites of the vitamin $\mathrm{D}_{3}$ : 24-hidroxicolecalciferol, 24,25-dihidroxicolecalciferol and 1,25 dihidroxicolecalciferol aforesaid as vitamin D3 total in 50 patients with osteopenia or osteoporosis. The trial was carried out for a system of HPLC. The percent recovery post-extraction went of the $85 \%$, the sensibility for the 1,25 dihidroxicolecalciferol went of $12.37 \mathrm{pg} / \mathrm{ml}$ and of the colecalciferol of $25 \mathrm{pg} / \mathrm{ml}$. The average of Vitamin $\mathrm{D}_{3}$ total went of $2072 \mathrm{ng} /$ $\mathrm{ml}$ with an interval from 6.3 to $10600 \mathrm{ng} / \mathrm{ml}$ and was found differentiates significant in the Vitamin D concentration averages among women with DMO normal and the ones that had al less osteopenia in hip $(p<0.04)$, nevertheless this difference was not significant in column. A significant difference of average concentrations of vitamin was found $D$ among cases with and without antecedent of fracture $(p=0.12)$. The relation of the concentrations of vitamin is discussed D with the bony mineral values of density and sociodemographic, biological, and clinical variables.

Key words: Vitamin $\mathrm{D}_{3}$ and metabolites, HPLC, Cartridges Sep-Pak, Colecalciferol, 1,25-(OH) $-\mathrm{D}_{3}$, Osteoporosis.

\section{Introducción}

La vitamina $\mathrm{D}$ es la única prohormona (estiroidea) que se encuentra en la sangre en dos distintas formas químicas: la vitamina $\mathrm{D}_{2}$ (Ergocalciferol), de origen vegetal, y la vitamina $\mathrm{D}_{3}$ (Colecalciferol), sintetizada en la piel u obtenida en la dieta alimenticia (1-7). Actualmente, cuando hablamos de vitamina D nos estamos refiriendo a la vitamina $\mathrm{D}_{3}$, más utilizada y que tiene un mayor espectro de aplicación (8-11). El 7-dehidrocolesterol, gracias a la acción de la luz ultravioleta origina la vitamina $\mathrm{D}_{3}$; este metabolito, si bien es el más abundante en el plasma, es biológicamente inerte. Su metabolito activo primario y la forma hormonal es el 1,25-dehidrocolecalciferol $\left[1,25-(\mathrm{OH})_{2} \mathrm{D}_{3}\right]$ o Calcitriol, el cual es producto de dos hidroxilaciones sucesivas en la molécula de Colecalciferol (9). La vitamina $\mathrm{D}$ es ampliamente reconocida como una hormona $(1,9,12) 1,25-(\mathrm{OH})_{2} \mathrm{D}_{3}$ como tal, se sintetiza en el organismo, es transportado en sangre, se activa en ciertos órganos y ejerce sus efectos biológicos interactuando con sus receptores específicos localizados en diversas células blancos (10). Además, su producción y degradación son procesos regulados por mecanismos de retroalimentación que responden a factores iónicos $\left(\mathrm{Ca}^{++}, \mathrm{P}\right)$, polipeptídicos (PTH, calcitonina), 1,25- $(\mathrm{OH})_{2} \mathrm{D}_{3}$ (esteroideos) y, probablemente, también por la acción de la prolactina y los estrógenos (12). Los órganos blancos principales del 1,25- $(\mathrm{OH})_{2} \mathrm{D}_{3}$ son el riñón, el hueso y el intestino, cuya acción fisiológica va dirigida a la absorción, mantenimiento y homeostasis de los iones minerales de calcio, fósforo y otros, siendo un factor importante en el crecimiento (1-3) y endurecimiento de huesos $(4,5,8)$ y dientes $(6,7,12)$. Así mismo, se han identificado receptores específicos para la forma activa de la vitamina $\mathrm{D}$ en una amplia variedad de tejidos (13-15). Esta importante sustancia migra a un elevado número de tejidos diana donde tienen lugar interacciones específicas responsables de un conjunto significante de respuestas biológicas (14) como la regulación de la transcripción de ciertos genes, promoción de la diferenciación celular $(16,17)$, regulación de la proliferación de células normales y malignas, estimulación del sistema inmunológico (1, $4,11)$.

www.unicolmayor.edu.co 
Actualmente, la vitamina D está relacionada con varias entidades de interés a nivel general como son osteoporosis $(18,19)$, raquitismo $(1,7,18)$, osteomalacia $(12,19)$, osteodistrofia renal, retinopatía severa en pacientes diabéticos (17), psoriasis (16), cáncer, leucemia (15), arritmias cardiacas (4), escleroderma, SIDA y enfermedades neurodegenerativas (11), que han motivado el desarrollo de investigaciones que ayuden a la prevención y tratamiento e incluyen la síntesis de nuevos fármacos para aplicaciones clínicas.

La osteoporosis es una condición metabólica del tejido óseo que conduce a una pérdida progresiva de la matriz mineral con la subsecuente fragilidad y predisposición a fracturas, considerada desde el punto de vista genético como un modelo multifactorial. En la actualidad, constituye un grave problema médico que tiene además una importante repercusión social y que presenta gran incidencia entre las mujeres especialmente a partir de los 50 años $(20,21)$. Riggs y Melton clasificaron la osteoporosis primaria en dos diferentes síndromes clínicos: Tipo I u osteoporosis postmenopáusica y Tipo II u osteoporosis senil relacionada con la edad. En el mundo se calculan 200 millones de personas afectadas. En Latinoamérica, 12.5 millones de personas son víctimas de fracturas.

En los trabajos realizados por el Instituto Nacional de Salud (INS) en Bogotá con una muestra estratificada de mujeres por encima de los 40 años y pertenecientes a diferentes orígenes, se encontró que la osteoporosis aumenta en forma notoria y acelerada entre las décadas quinta y sexta de la vida, con una de las prevalencias más altas en relación con las estadísticas internacionales para estos grupos etarios. El estudio de prevalencia de osteoporosis del INS mostró que al menos el $57 \%$ de la población mayor de 60 años tiene osteoporosis y se proyecta que medio millón de personas la tendrían para el año 2001 (23). La osteoporosis es una condición en incremento (21). Estudios de Medellín y Barranquilla demuestran frecuencias muy similares a las presentadas a nivel internacional $(22,23)$.

Actualmente hay gran interés en el metabolismo mineral y el papel de la vitamina $\mathrm{D}_{3}$, por su importancia en el entendimiento de la patogénesis de la osteoporosis y, en consecuencia, por sus aplicaciones terapéuticas y preventivas (24). A causa de los cambios en la circulación del 1,25- $(\mathrm{OH})_{2} \mathrm{D}_{3} \mathrm{y}$ su influencia en la fisiopatología de enfermedades del metabolismo del calcio se debe hacer el esfuerzo de desarrollar ensayos o métodos que permitan determinar este importante analito en plasma, siendo una ventaja conocer el status de vitamina D de los individuos antes de una prescripción terapéutica (9). El método por el sistema HPLC es altamente sensible y específico, que a diferencia de otras metodologías no requiere radioisótopos que puedan poner en riesgo la salud del operario, su entorno y el medio ambiente. Dado que la vitamina D se encuentra presente en cantidades muy pequeñas en los fluidos biológicos y por sus características lipídicas, la cuantificación de la Vitamina D implica una metodología compleja de varios pasos: separación, extracción, fraccionamiento y cuantificación; por lo tanto, requiere el desarrollo de métodos diversos buscando precisión $(25,29)$ y versatilidad (26). En la última década, hay interés por estudiar los metabolitos de la vitamina $\mathrm{D}$, en particular si están relacionados con la acción hormonal del 1,25$(\mathrm{OH})_{2} \mathrm{D}_{3} \mathrm{y}$ el metabolito intermediario $25-\mathrm{OH}-\mathrm{D}_{3}$. La mayoría de las técnicas para tal fin involucran el HPLC. Sin embargo, los procedimientos de extracción usados algunas veces se basan en métodos convencionales que incluyen requerimiento de grandes volúmenes de muestra, formación de emulsiones y recuperación errada de compuestos no deseados, siendo ineficientes en la remoción de sustancias químicamente similares a la vitamina D. Con el presente estudio se pretende planear, diseñar y ejecutar todos los procedimientos necesarios para llevar a cabo la estandarización de la metodología para 
determinar la vitamina D en Colombia y cuantificar las concentraciones séricas de los principales metabolitos de la vitamina $\mathrm{D}_{3}$ : 24-hidroxicolecalciferol, 24,25-dihidroxicolecalciferol y 1,25 dihidroxicolecalciferol, expresados como vitamina $\mathrm{D}_{3}$ total, principalmente en pacientes con riesgo de osteoporosis, considerando su posible utilidad para detectar precozmente esta condición, factible de prevención médica sustitutiva. Dicho ensayo está basado en un sistema de HPLC, la extracción y purificación en fase sólida, utilizando cartuchos de silica $\mathrm{C}_{18}$ (SepPak), que permite obtener extractos eluidos mediante solventes orgánicos, los cuales contienen el analito que posteriormente se cuantifica.

\section{Materiales y métodos}

Obtención de sueros humanos: Venopunción a 50 pacientes voluntarios de sexo femenino procedentes de varios municipios del Departamento de Cundinamarca en Colombia, con osteopenia u osteoporosis (documentado por DMO Densitometría Mineral Ósea), asistentes a consulta externa en el Hospital de La Samaritana y el centro médico Salud Reinun en Bogotá D.C. Se centrifugó la muestra de sangre sin anticoagulante entre 2.500 y 3.000 r.p.m. durante 15 minutos y se conservaron en congelación a $-20^{\circ} \mathrm{C}$ hasta el momento de iniciar la determinación.

Extracción de la muestra: A 0.2 a $1.0 \mathrm{ml}$ de suero en un tubo eppendorf, se agregó un volumen de acetonitrilo, se agitó vigorosamente en un vórtex por 20 segundos y se llevó a centrifugación $\left(20^{\circ} \mathrm{C}, 2.000\right.$ $\mathrm{x} g, 10$ minutos). Después de centrifugada se decantó el sobrenadante dentro de un tubo de vidrio 13100 que contenía 0.5 volumen de $\mathrm{K}_{2} \mathrm{HPO}_{4} 0.4 \mathrm{M}$ pH 10.5 y se mezcló en un vórtex por 30 segundos; luego se centrifugó a 2.000 gravedades por 10 minutos a $20^{\circ} \mathrm{C}$.

Purificación de la Vitamin a D y sus metabolitos: El extracto obtenido se aplicó directamente al cartucho Sep-pak Plus $\mathrm{C}_{18}$ de asociación Waters, previa- mente activado, y se lavó con $5 \mathrm{ml}$ de agua desionizada y $3 \mathrm{ml}$ de metanol: agua (70:30). Estas dos fracciones se descartaron, se eluyó con $3 \mathrm{ml}$ de acetonitrilo (fracción recolectada), se secó la fracción obtenida bajo flujo de nitrógeno y resuspendió con $0.5 \mathrm{ml}$ de hexano: isopropanol (96:4), al cartucho de silica $\mathrm{C}_{18}$ Sep-Pak Plus se aplicó el extracto que contiene los metabolitos de la Vitamina D, lavando el tubo con la adición de $0.5 \mathrm{ml}$ más de solvente, se lavó sucesivamente con $11 \mathrm{ml}$ de hexano: isopropanol (96:4), $8 \mathrm{ml}$ de hexano: isopropanol (94:6) y con $10 \mathrm{ml}$ de hexano: isopropanol (85:15), se recolectaron las fracciones y secaron bajo flujo de nitrógeno.

Procedimiento de activación de los cartuchos Sep-Pak C ${ }_{18}$ : Los cartuchos nuevos y los reutilizados deben ser activados o prelavados para su uso con la adición secuencial de $5 \mathrm{ml}$ de hexano, $5 \mathrm{ml}$ de cloroformo, $5 \mathrm{ml}$ de metanol y $5 \mathrm{ml}$ de agua.

Procedimiento de lavado de los cartuchos SepPak $\mathrm{C}_{18}$ : Los cartuchos utilizados fueron lavados con la adición secuencial de $5 \mathrm{ml}$ de metanol, $5 \mathrm{ml}$ de cloroformo, $5 \mathrm{ml}$ de hexano y $5 \mathrm{ml}$ de hexano-isopropanol (96:4).

Cuantificación de la Vitamin a D y sus metabolitos: Las fracciones recolectadas fueron resuspendidas en 50 ul. de metanol o etanol e inyectadas al equipo para HPLC utilizando: Fase Móvil Acetonitrilo: Metanol: Agua 25:25:1, solventes grado HPLC y pasados por desgacificador con filtros de membrana de Millipore ${ }^{\circledR}$ de 0.5 , longitud de onda de $265 \mathrm{~nm}$ con 486 Tunable Absorbance Detector: Waters ${ }^{\circledR}$, tiempo de corrida 10 minutos, fase reversa con columna Simmetry ${ }^{\circledR} \quad \mathrm{C}_{8} 5 \mathrm{um}, 3.9$ x $150 \mathrm{~mm}$ Column Waters, TM y Controller Waters ${ }^{\circledR}$ TM 600. El estándar es directamente comparado con la muestra.

Cálculos: El graficador mediante el software Millenium muestra las concentraciones de Vitamina $\mathrm{D}_{3}$ y sus metabolitos $25-(\mathrm{OH}) \mathrm{D}_{3}, 24,25-(\mathrm{OH})_{2} \mathrm{D}_{3}$ y del 1,25$(\mathrm{OH})_{2} \mathrm{D}_{3}$ del suero en forma de pico cromatográfico. El 
área bajo la curva y/o la altura de cada pico es directamente proporcional a la concentración de la sustancia, lo cual permite la cuantificación precisa de ésta. Los datos se tabularon en Excel 97-4.0 y procesados en Epi Info 6 versión 6.04a.

\section{Resultados}

Caracterización cromatográfica de la vitamina D: Se compararon las columnas NovaPak y Simmetry para definir cuál de estas permite una mejor resolución de las vitaminas liposolubles. Para ello, se utilizaron los estándares de trans-Retinol (vitamina A) y Colecalciferol (vitamina D). Dentro de las características cromatográficas, la columna Simmetry permitió mayor resolución de dichas sustancias. Adicionalmente se evaluaron los estándares de Uracilo y alfa-Tocoferol como estándares internos de la columna, determinando la utilización del Uracilo para estabilizar la columna, mas no como estándar interno a bajas concentraciones.

El estándar de trans-Retinol presentó varios picos. Los estándar de Colecalciferol y alfa-Tocoferol son los que reportaron picos más definidos, agudos con bases simétricas aún en varias diluciones. La mayor absorción para la vitamina $\mathrm{D}$ se hace en una longitud de onda de $265 \mathrm{~nm}$, según los ensayos realizados desde 254 a $280 \mathrm{~nm}$. Con la fase móvil utilizada se logró una velocidad de corrida por muestra de $1.0 \mathrm{ml} / \mathrm{min}$ por 10 minutos. Los tiempos de retención obtenidos se muestran en la Tabla 1.

\begin{tabular}{lc} 
Vitamina & Tiempo de retención $(\mathrm{min})$ \\
\hline Uracilo & $1.3-1.5$ \\
trans-Retinol & 2.4 \\
Colecalciferol & $4.5-5.0$ \\
alfa-Tocoferol & $5.5-6.0$ \\
Calcitriol & 3.5 o 5.5 \\
\hline
\end{tabular}

Tabla1. Tiempos de retención de las vitaminas liposolubles que han sido descritas por algunos autores como interferentes de los análisis para cuantificar Vitamina D; y del Uracilo postulado para ser utilizado como estándar interno.

Recuperabilidad : Dado que para extraer la vitamina $\mathrm{D}$ se requiere de un proceso largo y un tanto dispendioso, se consideró importante calcular qué por- centaje se recupera de la vitamina tras su paso por el cartucho de silica $\mathrm{C}_{18}$, recuperándose el $85 \%$ si se hace elusión con acetonitrilo y el extracto previo eluido con metanol únicamente contendría el 15\% restante.

Curva de calibración: La curva de calibración para cuantificar vitamina $D$ total se realizó con el área bajo la curva versus concentración y se estableció con estándares certificados de 1,25- dihidrocolecalciferol y colecalciferol, obteniéndose un coeficiente de regresión lineal $\mathrm{Re}=0.9932\left(\mathrm{R}^{2}=0.9864\right)$.

Concentración de Vitamina D: La concentración media de vitamina $\mathrm{D}_{3}$ total en muestras séricas de nuestro estudio fue de $2072 \mathrm{ng} / \mathrm{ml}$, con un intervalo de 6,3 a $10600 \mathrm{ng} / \mathrm{ml}$. La mayoría de las concentraciones estuvieron por debajo de los $4 \mathrm{pg} / \mathrm{ml}$, como muestra la figura 1, y la mayor frecuencia de estas concentraciones estuvo entre 1 - $2 \mathrm{pg} / \mathrm{ml}$ como se muestra en la Figura 2. La sensibilidad lograda hasta el momento es del orden de $2 \mathrm{pg}$. por inyección que equivalen a $40 \mathrm{pg} / \mathrm{ul}$. Los cromatogramas obtenidos para los principales metabolitos de la vitamina D se muestran en la Figura 3.

Finalmente, la comparación de las medias de las concentraciones de la vitamina $\mathrm{D}$ entre las mujeres normales y con osteoporosis u osteopenia, según su DMO, demostró que existe una diferencia estadísticamente significativa en las medias de concentración de Vitamina D entre mujeres con DMO normal $(\mathrm{p}<0.04)$ y las que tenían al menos osteopenia en cadera (Tabla 2), pero esa diferencia no fue significativa en columna (Tabla 3 ). La vitamina D tiene un efecto sobre la frecuencia de fracturas, aunque los informes publicados son muy heterogéneos. Se halló una diferencia significativa de concentraciones medias de vitamina $\mathrm{D}$ entre casos con y sin antecedente de fractura $(\mathrm{p}=0.12)$, según muestra la Tabla 4.

www.unicolmayor.edu.co 


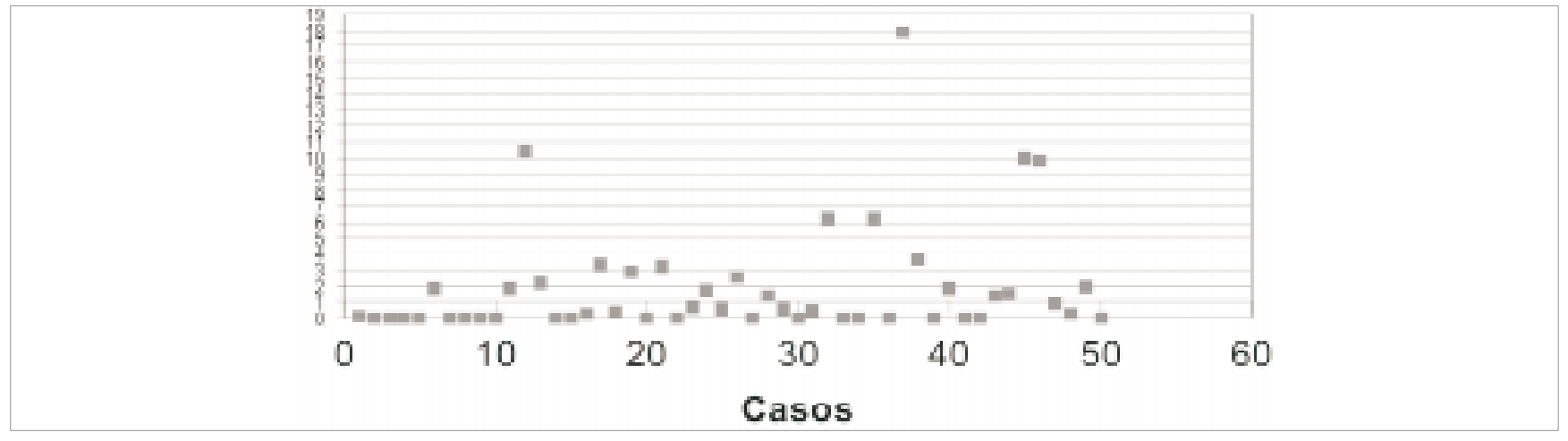

Figura 1 . Dispersión de la concentración de vitamin a D y casos. La concentración media de vitamina D3 total en suero fue de 2072 ng/ml, intervalo de 6,3 a $10600 \mathrm{ng} / \mathrm{ml}$.

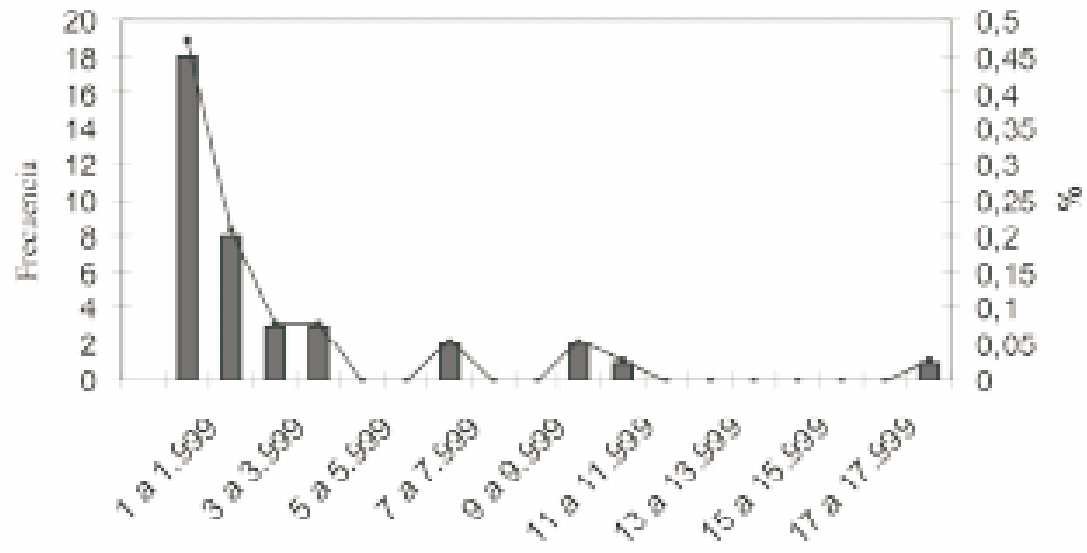

Figura 2 . Distribución de frecuencias de concentraciones séricas de vitamina D.

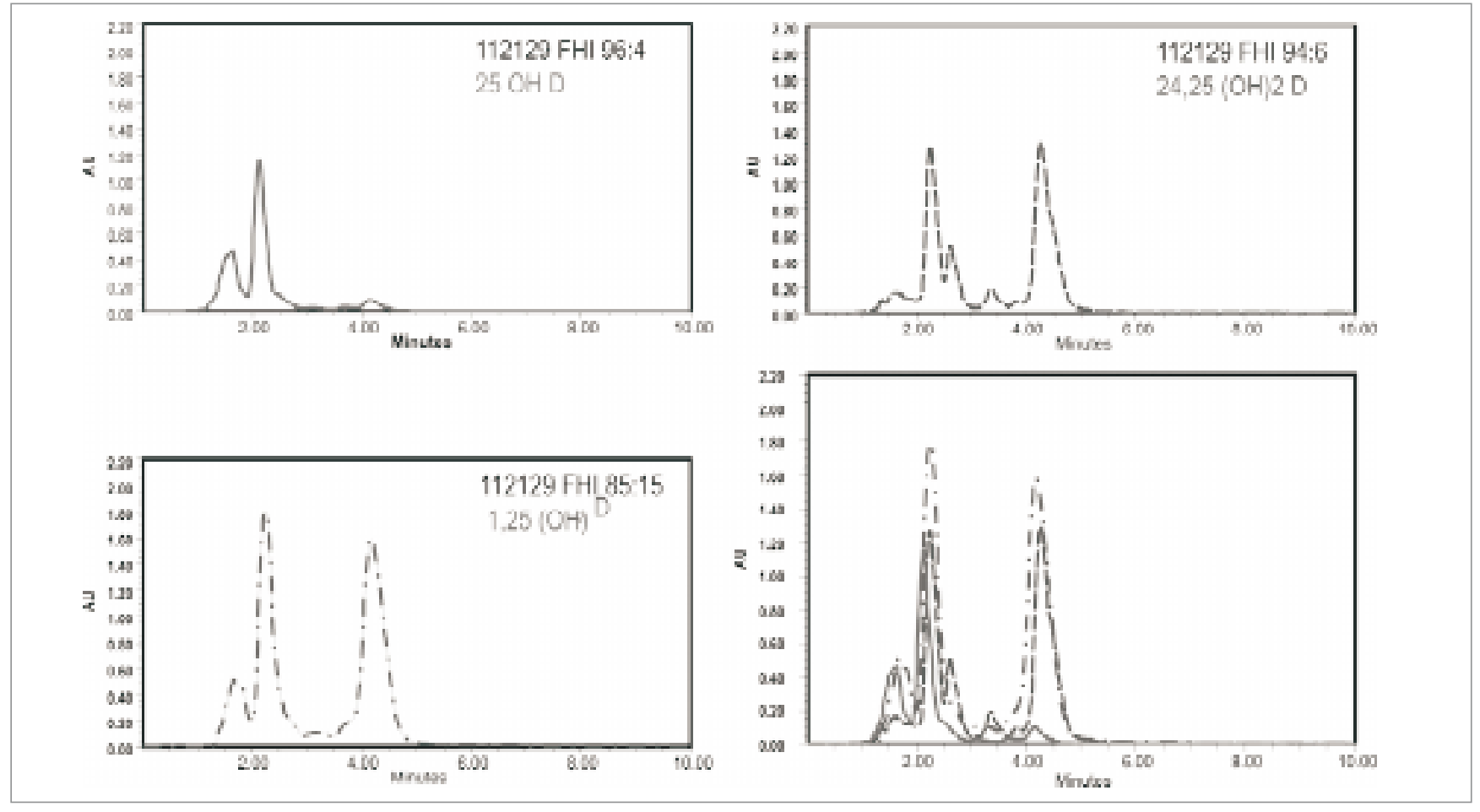

Figura 3. La caracterización cromatográfica de los principales metabolitos de la Vitamina D.Los cromatogramas de los principales metabolitos de la Vitamina D extraídos con hexano- isopropanol. Sensibilidad de $12.37 \mathrm{pg} / \mathrm{ml}$ para 1,25-dihidroxivitamina D3, y 25 pg/mL para vitamina D3. 
Cadera

F

$\mathrm{p}^{*}$

Densitometría compatible con:

\begin{tabular}{lll} 
Osteopenia Vs. Osteoporosis & 0,38 & 0,542 \\
Osteopenia u Osteoporosis Vs. Normal & 4,22 & 0,0478 \\
Osteopenia Vs.Normal & 2,54 & 0,1261 \\
Osteoporosis Vs.Normal & 1,14 & 0,48 \\
* Significativo p<0.1 & & \\
\hline
\end{tabular}

Tabla 2. Comparación de Medias de Concentración de Vitamina D según a condición de Densidad Ósea de Cadera

\begin{tabular}{|c|c|c|}
\hline Columna & $\mathrm{F}$ & $\mathrm{p}^{*}$ \\
\hline \multicolumn{3}{|l|}{ Densitometría compatible con: } \\
\hline Osteopenia Vs. Osteoporosis & 0,2 & 0,6595 \\
\hline Osteopenia u Osteoporosis Vs. Normal & 0,17 & 0,6866 \\
\hline$*$ Significativo $\mathrm{p}<0.1$ & & \\
\hline
\end{tabular}

Tabla 3. Comparación de Medias de Concentración de Vitamina D según a condición de Densidad Ósea de Columna

\begin{tabular}{lll} 
Fractura previa & $\mathrm{F}$ & $\mathrm{p} *$ \\
\hline Sí Vs. No & 2,55 & 0,128 \\
* Significativo $\mathrm{p}<0.1$ & & \\
\hline
\end{tabular}

Tabla 4. Comparación de Medias de Concentración de Vitamina D según antecedentes de fractura Previa.

\section{Discusión}

En sus estudios sobre cromatografía, H.R. Bolliger señala la vitamina A como un interferente cuando se quiere determinar Vitamina D (30), debido a que ambas vitaminas son liposolubles y están presentes en el suero; además, sugiere la utilización de columnas como fase estacionaria en un proceso de cromatografía. Por tanto, se evaluó la resolución entre las columnas NovaPak y Simmetry, con los estándares trans-Retinol (vitamina A) y Colecalciferol (Vitamina D), demostrando que la columna Simmetry tuvo mejor resolución para estos dos estándares y se utilizó durante la metodología del estudio. Los cromatogramas del estándar de trans-Retinol presentaron varios picos, esto puede deberse a la presencia de isomeros, posiblemente por la mayor sensibilidad de la vitamina a variación con los rayos solares y la luz en general. Sin embargo, se estableció que el Retinol y su tiempo de retención no interfieren con la vitamina D. El uracilo se escogió como estándar interno puesto que su estructura química corresponde a un anillo pirimidínico, anillo que hace parte también de la estructura de la vitamina $\mathrm{D}$, posee absorción a la misma longitud de onda en que absorbe el analito de interés, no se encuentra normalmente en fluidos corporales y, aunque se comparte alguna similitud estructural, tienen diferente comportamiento cromatográfico. Sin embargo, cuando se determinó a qué concentraciones mínimas el uracilo puede ser utilizado como estándar interno, ocurre un fenómeno de traslapación de picos o picos de bases unidas, por tanto este estándar sirvió para estabilizar la columna pero no se utilizó como estándar interno a bajas concentraciones. Los estándares Colecalciferol y alfaTocoferol son los que reportaron picos más definidos, agudos, con bases simétricas aún en varias diluciones. El estándar de alfatocoferol también se escogió como estándar interno. La mayor absorción del Colecalciferol fue a una longitud de onda de $265 \mathrm{~nm}$, según los ensayos realizados en el rango de 254 a $280 \mathrm{~nm}$. La velocidad de corrida por muestra es de $1.0 \mathrm{ml} / \mathrm{min}$. de fase móvil durante 10 minutos. Los estándares que se usaron no son marcados con isótopos; por tanto, representan menor riesgo en el manejo y tienen poco impacto ambiental que los citados en otras metodologías, RIA e IRMA. A medida que se conocieron las características cromatográficas de cada una de las sustancias utilizadas como estándar, se hizo posible la adecuada identificación de la vitamina $\mathrm{D}$ dentro de una muestra problema.

Teniendo en cuenta que la vitamina $\mathrm{D}$ y sus analitos se encuentran en bajas concentraciones en suero y que su origen es lipídico, se utilizaron cartuchos de sílica $\mathrm{C}_{18}$ SepPak, lográndose una eficiente extracción de todos los componentes de interés y corroborando que se puede lograr extracción en fase sólida como lo expuesto por Hollis y Frank (29). En cuanto a la extracción y purificación, consideramos que hay una excelente recuperabilidad del estándar de Colecalciferol, con un porcentaje del $85 \%$, tras el procesamiento inicial y su paso por el cartucho, este por- 
centaje se puede mejorar utilizando mayor volumen de muestra. El material lipofílico extraído fue evaluado por cromatografía líquida de alta resolución (HPLC), con columna de silica $\mathrm{C}_{8}$, técnica efectiva en la resolución de la Vitamina D y sus metabolitos por incremento de la polaridad; por esta razón la fase móvil se compone de acetonitrilo, metanol y agua con polaridad ascendente. La evaluación de la repetibilidad de los procesos arrojó resultados esperados, lo que contribuye confiabilidad de resultados y coordinación adecuada entre el equipo instrumental y humano.

La curva de calibración se realizó con el área bajo la curva versus concentración. No se realizó absorbanciaconcentración porque en un cromatograma no siempre la máxima absorción representa su equivalente, en área bajo la curva. El área bajo la curva y/o la altura de cada pico cromatográfico es directamente proporcional a la concentración de la sustancia, lo cual permite la cuantificación precisa de ésta. La curva de calibración que se utilizó como parámetro de control de calidad interno y cuantificación de la vitamina $\mathrm{D}$ se obtuvo con una regresión lineal de $0.99\left(\mathrm{R}^{2}=0.98\right)$.

La sensibilidad es del orden de 2 pg por inyección que equivalen a $40 \mathrm{pg} / \mathrm{ul}$; es una alta sensibilidad que también puede corregirse utilizando más volumen de muestra, menor volumen de reconstituido e incrementar el volumen de inyección de 20 a 100 ul; la sensibilidad es óptima, si se tiene en cuenta que normalmente la concentración de la vitamina D en muestras biológicas es baja, se trabaja en el orden de picogramos y algunas patologías como osteoporosis pueden cursar con hipovitaminosis (31).

En la comparación de la media de las concentraciones de vitamina $\mathrm{D}$ de poblaciones clasificadas según su condición de DMO para cadera y columna, existe una diferencia significativa en las medias de vitamina $\mathrm{D}$ entre mujeres con densidad mineral ósea normal y las que tenían al menos osteopenia en cadera $(\mathrm{p}<0,04)$ (Tabla 2$)$, pero esa diferencia no fue significativa en columna (Tabla 3 ), lo que significa que el metabolismo óseo es diferente en estas dos regiones óseas; además, indica que los niveles de Vitamina D son más utilizados para predecir osteopenia y osteoporosis en cadera que en columna. Como se observa en la Tabla 4, se encontró una diferencia significativa al nivel las concentraciones medias de vitamina D entre casos con y sin antecedente de fractura $(\mathrm{p}=0,12)$, que señalarían la utilidad de la terapia con vitamina $\mathrm{D}$ en pacientes con riesgo de fractura, aunque los informes publicados al respecto son contradictorios.

A pesar de la complejidad en el procesamiento de la muestra, es útil estandarizar métodos para cuantificar vitamina D por su importancia en el metabolismo mineral y su relación con otras variables clínicas, biológicas y sociodemográficas $(32,33)$.

Se concluyó que la metodología de HPLC tiene una aplicabilidad ventajosa para la cuantificación de Vitamina D, puesto que permitió el manejo simultáneo de múltiples muestras en un tiempo razonable. En comparación con otros métodos, los beneficios que hasta ahora se han logrado con este método son alta sensibilidad y especificidad. Por otra parte, se resalta que la cuantificación de Vitamina D es importante en personas con osteopenia, bien sea como indicador de riesgo de fractura, así como estrategia para orientar el tratamiento farmacológico con vitamina $\mathrm{D}$, puesto que la osteoporosis es una condición metabólica crónica, cuya secuela más grave y más frecuente es la fractura. Esta patología avanza como una epidemia silenciosa que afecta a hombres y mujeres, disminuye la calidad de vida, incrementa el riesgo de mortalidad y constituye un gran problema de salud pública. Se necesita hacer una medición poblacional de Vitamina D en población sana para disponer de valores de referencia para Colombia; de allí la importancia del desarrollo de metodologías que permitan una alerta y un tratamiento oportuno porque un diagnóstico temprano puede evitar fracturas y todas sus consecuencias devastadoras. 


\section{Referencias}

1. URL:http://www.medspain.com/ant/n14_jul00/vit.htm

2. Ilczyszyn GR, Gurí JC. Las virtudes de la vitamina D contra la osteoporosis. 1999. Disponible en: URL: http:// www.healthig.com/vitaminas/vitaminas4.html

3. Rubio LE, Morán M, Talbot JR, ZanchettA JR. Concentración sérica de 25 (ho) vitamina $\mathrm{D}$ en sujetos jóvenes y añosos residentes en buenos aires Trabajo Original. The Official Journal of the Pan American Osteoporosis Foundation, Instituto de Investigaciones Metabólicas (IDIM), Universidad del Salvador. Disponible en: URL: http://www.osteored.com.ar/web/osteored.nsf

4. La vitamina D y sus principales funciones. 1999. Disponible en: URL: http://www.forever-life.com/vitaminaD.htm

5. Koller T. La Vitamina D: el «beso del sol» para nuestra salud, bloqueada por la computadora!. 2000. Disponible en: URL: http://cuerpodiet.com/tusvitaminas/vitaminaD.htm

6. En buenas manos. Vitamina D. Portal de salud y terapias naturales. Disponible en: URL: http://www.enbuenasmanos.com/ARTICULOS/muestra.asp? art $=351$ \&unic $=1$

7. La importancia de la vitamina D en los recién nacidos. Salud. Disponible en: URL: http://www.zonadiet.com/salud/vit-dlactancia.htm

8. University of Glasgow. 1,25 Dihydroxy Vitamin D. 1996. Disponible en: URL: http://www.pdg.cnb.uam.es/cursos/Barcelona2002/pages/Farmac/Immunoassays/125DWeb.htm

9. Timothy A, et al. A Microassay for 1,25-Dihydroxyvitamin D Not Requiring High Performancen Liquid Chromatography: Application to Clinical Studies. Journal of Clinical Endocrinology and Metabolismo 1984;58(1):91-8.

10. Díaz L, Cariño C, Méndez I. La vitamina D: implicaciones en la salud y el embarazo. Revista de Investigación Clínica 2001;53(1):77-85.

11. Ferrero M, Fernández S. Vitamina D y terapias contra las enfermedades neurodegenerativas químicos del principado. 1994;(34). Colegio Oficial de Químicos de Asturias y León 2000. Disponible en: URL: http://www.alquimicos.com/quimprin/34/ vitamina.html.

12. Beyond discovery. Para aclarar el enigma de la vitamina D. Vínculo de la vitamina D con el control del calcio. Disponible en: URL: http://www7.nationalacademies.org/ spanishbeyonddiscovery/bio_008721-06.html

13. Cravioto MdC. Vitamina D placentaria: síntesis, regulación e implicaciones clínicas. Revista de Investigación Clínica 2000;52(6):692-4.

14. Mouriño Mosquera JA, et al. Diseño, síntesis e implicaciones biológicas y terapéuticas de nuevos análogos de la vitamina D. Facultad de Química, Santiago de Compostela. 15706 Disponible en: URL: http://qoweb.usc.es/investigacion/grupos/AM/ indice.html

15. Mangelsdorf DJ, Evans R. La vitamina D sería fundamental para prevenir el cáncer de colon. Instituto Médico Howard Hughes (HHMI). Science 2002. Disponible en: URL: http:// www.hhmi.org/news/mangelsdorf2-esp.html.

16. Derivados vitamina D. Psoriasis. Disponible en: URL: http:// www.humano.ya.com/psoriasis/VitaminaD.htm11.Noticias sobre la vitamina D.
17. Taverna MJ, et al. Taq I polymorphism of the vitamin D receptor and risk of severe diabetic retinopathy. Diabetología 2002;45:436-42.

18. Noticias sobre la vitamina D. Disponible en: URL: http:// www.dietasalamedida.com/todito/noticias/ noticias_sobre_la_vitamina_d.asp.

19. Faes farma. Área de comunicación desarrollo de noticia. Symposium satélite sobre actualizaciones en la clínica geriátrica: depresión/osteoporosis. Disponible en: URL: http:// www.faes.es/area comunicación/desarrollo noticia/ 010200.html.

20. Fonendo. Nutrición. A la vejez... nueces!!! Disponible en: URL: www.fonendo.com/noticias/40/2000/09/5.shtm.

21. Asociación Colombiana de Osteología y Metabolismo Mineral. Asociación Colombiana de Osteoporosis (ACOMM). Conferencia de consenso diagnóstico y manejo de la osteoporosis. Congreso Colombiano de Osteoporosis; 2001. Disponible en: URL: http:/www.paof.org/consensos11.pdf

22. Ardila E. Características de la Osteoporosis en Colombia. Revista de la Asociación colombiana de Gerontología y Geriatría 2002;16(3).

23. Ching Pontón RB, et al. Factores de riesgo para osteoporosis y osteopenia en 707 mujeres seleccionadas en Bogotá. Reemo $2001 ; 10(3)$.

24. Prockop D. The genetic trail of osteoporosis. The New England Journal of Medicine 1996;338(15):1061-2.

25. Hollis BW, Kamerud JQ, Kurkowski A, Beaulieu J, Napoli JL. Quantification of circulating 1,25-dihydroxivitamin D by radioinmunoassay with an I 25I-labeled tracer. Clinical Chemistry 1998;42(4):586-92

26. Koyama H, Prahl JM, Uhland A, Nanjo M, Inaba M, et al. A New, Highly Sensitive Assay for 1,25-Dihyroxyvitamin D Not Requiring High-Performance Liquid chromatography: Application of Monoclonal Antibody against Vitamin D Receptor to Radioreceptor Assay. Analytical Biochemistry 1992;205:213-9

27. Hollis BW. Assay of Circulating 1,25-Dihydroxyvitamin D Involving a Novel Single- Cartridge Extraction and Purification Procedure. Clinical Chemistry 1986;32(11):2060-3.

28. P. Universidad Católica de Chile. Osteomalacia. Apuntes de reumatología. Disponible en: URL: http://escuela.med.puc.cl/ publ/reumatologia/Apuntes/16Osteomalacia.html

29. Hollis, B y Frank, N. Solid phase extraction system for d and its major metabolites in human plasma. Journal of Chromatography. Biomedical Applications 1985;343:43-9.

30. Bolliger, H.R. En: Stahl E. Mun-layre Chromatography. Springen Verlag, Berlín, Heidelberg, New York, Londres: Academic Press. Inc.; 1965. p. 227-8

31. Favus MJ. Primer on the Metabolic Bone Diseases and Disorders of Mineral Metabolism. 2nd ed. New York: Raven Press; 1993.

32. Heaney Rp. Bone Mass, Nutrition and other lifestyle factors. The American Journal of Medicine 1993;95(Suppl 5a):29s-31s.

33. Delmas PD. Biochemical Markers of Bone turnover I: Theoretical Considerations and Clinical Use in Osteoporosis. The American Journal of Medicine 1993;95(Suppl 5):11-6. 